\title{
Differentially Expressed miRNAs in Tumor, Adjacent, and Normal Tissues of Lung Adenocarcinoma
}

\author{
Fei Tian, ${ }^{1}$ Rui Li, ${ }^{1}$ Zhenzhu Chen, ${ }^{1}$ Yanting Shen, ${ }^{1}$ Jiafeng Lu, ${ }^{2}$ \\ Xueying Xie, ${ }^{1}$ and Qinyu $\mathrm{Ge}^{1,2}$ \\ ${ }^{1}$ Research Center for Learning Science, Southeast University, Nanjing 210096, China \\ ${ }^{2}$ State Key Lab of Bioelectronics, Southeast University, Nanjing 210096, China \\ Correspondence should be addressed to Qinyu Ge; geqinyu@seu.edu.cn
}

Received 24 December 2015; Revised 4 March 2016; Accepted 22 March 2016

Academic Editor: Melchiorre Cervello

Copyright (C) 2016 Fei Tian et al. This is an open access article distributed under the Creative Commons Attribution License, which permits unrestricted use, distribution, and reproduction in any medium, provided the original work is properly cited.

Lung cancer is the leading cause of cancer deaths. Non-small-cell lung cancer (NSCLC) is the major type of lung cancer. The aim of this study was to characterize the expression profiles of miRNAs in adenocarcinoma (AC), one major subtype of NSCLC. In this study, the miRNAs were detected in normal, adjacent, and tumor tissues by next-generation sequencing. Then the expression levels of differential miRNAs were quantified by quantitative reverse transcription-polymerase chain reaction (qRT-PCR). In the results, 259, 401, and 389 miRNAs were detected in tumor, adjacent, and normal tissues of pooled AC samples, respectively. In addition, for the first time we have found that miR-21-5p and miR-196a-5p were gradually upregulated from normal to adjacent to tumor tissues; miR-218-5p was gradually downregulated with 2-fold or greater change in AC tissues. These 3 miRNAs were validated by qRT-PCR. Lastly, we predicted target genes of these 3 miRNAs and enriched the potential functions and regulatory pathways. The aberrant miR-21-5p, miR-196a-5p, and miR-218-5p may become biomarkers for diagnosis and prognosis of lung adenocarcinoma. This research may be useful for lung adenocarcinoma diagnosis and the study of pathology in lung cancer.

\section{Introduction}

Lung cancer is the leading cause of cancer-related deaths in the world [1]. Non-small-cell lung cancer (NSCLC) is the main type of lung cancer, which accounts for $80-85 \%$. NSCLC mainly consists of squamous cell carcinoma (SCC) and adenocarcinoma (AC) [2]. Patients with NSCLC were frequently diagnosed at advanced stages, resulting in an overall 5-year survival rate of about $14 \%$ [3]. In order to improve the outcome of these patients, the early diagnosis of NSCLC becomes necessary. In recent years, studies showed that molecular biomarkers were discovered in various cancers and could be applied in the diagnosis and prognosis of NSCLC [4-6].

MicroRNAs (miRNAs) are endogenous noncoding small RNAs of about 18-25 nucleotides [7]. Mature miRNAs are highly conserved RNA molecules that can regulate the expression of genes by hybridizing to complementary sequences in the $3^{\prime}$-untranslated (UTR) region of target mRNAs [8]. Studies have shown that miRNAs participate in a lot of biological processes, including cell proliferation, cell differentiation, and cell apoptosis [9]. In addition, a large number of studies have shown that miRNAs may play roles in the tumorigenesis and development of lung cancer as tumor oncogenes or suppressor genes [10-14]. However, few researchers studied the expression profiles of miRNAs in paired tumor, adjacent, and normal tissues of lung cancer.

Three principal methods can be adopted to detect the expression levels of miRNAs: quantitative reverse transcription-polymerase chain reaction (qRT-PCR), microarray, and next-generation sequencing (NGS). Quantitative RT-PCR and microarray can only detect relative expression levels of known miRNAs, and the number of analyzed miRNAs is limited. Compared to qRT-PCR and microarray, NGS has an advantage in global miRNA analysis and it provides 
the possibility of detecting novel miRNAs. What is more, the analysis of miRNAs becomes faster and cheaper based on NGS [15].

In this study, the miRNAs were detected in tumor, adjacent, and normal tissues from AC pooled samples by NGS. The differential miR-21-5p, miR-196a-5p, and miR-218$5 \mathrm{p}$ were validated by qRT-PCR. The purpose of this study was to systematically characterize the expression of miRNAs in AC tumor, adjacent, and normal tissues. miR-21-5p, miR196a-5p, and miR-218-5p can be biomarkers for diagnosis and prognosis of AC. The gradually changed miRNAs may be useful for AC diagnosis and the study of pathology in lung cancer.

\section{Material and Method}

2.1. Sample Preparation and RNA Isolation. We collected 18 surgically resected samples including paired tumor, adjacent, and normal lung tissues from 18 AC patients. Tissue samples were all obtained from Jiangsu Province Hospital with informed consent. Tissues were stored at $-80^{\circ} \mathrm{C}$ and then ground for subsequent RNA isolation.

Total RNA was isolated from $50 \mathrm{mg}$ tissue of each sample by mirVana ${ }^{\mathrm{TM}}$ miRNA Isolation Kit (Ambion, USA). The quantity and quality of obtained RNA were measured by Qubit ${ }^{\circledR}$ 2.0 Fluorometer (Life Technologies, USA). The total RNA isolated from 9 AC tumor tissue samples was pooled with equimolar amounts, respectively, and the same operation was applied to $9 \mathrm{AC}$ adjacent and 9 normal tissues. The miRNAs of the 3 pooled samples were analyzed by nextgeneration sequencing. Another 9 paired AC samples were used for following qRT-PCR validation.

\subsection{Small RNA Library Preparation for Ion Torrent Sequenc-} ing. The small RNA libraries for Ion Torrent sequencing were prepared according to the manufacturer's protocols of Ion Total RNA-Seq Kit v2 (Life Technologies, USA) and Ion Xpress ${ }^{\mathrm{TM}}$ RNA-Seq Barcode 1-16 Kit (Life Technologies, USA). The libraries were sequenced by Ion Torrent Proton ${ }^{\mathrm{TM}}$ sequencing platform after quality control at Nanjing Percare Biotechnology Co., Ltd.

2.3. Bioinformation Analysis. The raw expression values (read counts) of Ion Torrent were filtered by quality control. The effective reads were analyzed through four steps. Firstly, the basic results were obtained, including the length distribution, mapping to reference genome, mapping to rfam, and the repeat distribution. Secondly, miRNAs (including novel miRNAs) were predicted. Thirdly, miRNA target genes were predicted and annotated. Lastly, other ncRNAs were annotated, including tRNA, rRNA, snoRNA, snRNA, and piRNA. Since low copy number was less reliable, the miRNAs with more than 10 copies were reserved for subsequent analysis. In order to compare miRNA expressions across databases, the total copy number of each sample was normalized to $1,000,000[16,17]$. According to some reports on multiple isomiRs from a given miRNA locus $[18,19]$, isomiRs were also comprehensively surveyed. Sequences that matched the premiRNAs in the mature miRNA region \pm 4 nt (no more than 1 mismatch) were defined as isomiRs.

2.4. Quantitative Validation of miRNAs by $q R T-P C R$. We performed quantitative RT-PCR (qRT-PCR) to validate the expression levels of miRNAs analyzed by NGS. The reverse transcription reaction was carried out with AMV reverse transcriptase (Takara, Japan) at $42^{\circ} \mathrm{C}$ for $60 \mathrm{~min}$ and $75^{\circ} \mathrm{C}$ for $15 \mathrm{~min}$ in a total volume of $20 \mu \mathrm{L}$. The universal RT primer was $5^{\prime}$-GTCGTATCCAGTGCAGGGTCCGAGGTATTCGCACTGGATACGACNNNNNN-3'. The quantitative PCR amplification was performed with SYBR Premix (Takara, Japan). The forward primers were miRNAs-specific, and the universal reverse primer was GTGCAGGGT CCGAGGT.U6 snRNA was used as internal reference. The reaction was incubated in ABI 7500 PCR system at $95^{\circ} \mathrm{C}$ for $5 \mathrm{~min}$, followed by 40 cycles of $95^{\circ} \mathrm{C}$ for $15 \mathrm{~s}$ and $55^{\circ} \mathrm{C}$ for $45 \mathrm{~s}$ in a total volume of $10 \mu \mathrm{L}$. The cycle threshold $(\mathrm{Ct})$ values were calculated by automatic Ct setting of 7500 system SDS software v1.4 (Applied Biosystems, USA).

2.5. Prediction and Functional Analysis of Target mRNA. We used the starbase v2.0 to predict the target gene of miRNAs verified by qRT-PCR $[20,21]$. The target genes were obtained by the intersection of three prediction software programs, which were TargetScan, PicTar, and miRanda based on the starbase. Then we used the Gene Ontology (GO) database for function enrichment [22, 23]. In addition, DNA intelligent analysis (DIANA) software was used for pathway identification based on Kyoto Encyclopedia of Genes and Genomes (KEGG) database [24].

2.6. Statistical Analysis. We adopted the $\log _{2}$ method to transform the normalized reads, and the expression patterns of the sequencing data were visualized by hierarchical clustering. The $p$ value of differential expression of miRNAs was calculated based on Poisson's distribution [25] and the threshold of $p$ value was determined by false discovery rate (FDR) [26]. The average $\mathrm{Ct}$ for each triplicate from qRT-PCR was used for analysis. Fold change in miRNA expression was calculated by $\Delta \Delta \mathrm{Ct}$, normalized with $\Delta \mathrm{Ct}=\mathrm{AvgCt}_{\text {miRNA }}-\mathrm{AvgCt}_{\mathrm{U} 6}$ [27]. A comparison between two groups was performed by using the $t$-test. $p$ values of $<0.05$ were considered to indicate statistically significant differences.

\section{Results}

3.1. Results of miRNA Sequencing. Based on Ion Torrent sequencing platform, the total RNAs of the 3 pooled samples from 9 paired $\mathrm{AC}$ tissues were characterized. In each pooled sample, more than $50 \%$ reads were annotated to miRNAs and ncRNAs database (data not shown). In this study, we detected 259, 401, and 389 known miRNAs in AC tumor, adjacent, and normal tissues, with 65,95 , and 34 novel mature miRNAs detected in the 3 pooled samples, respectively (Table 1). In addition, the sequences mapped with reference genome, on 


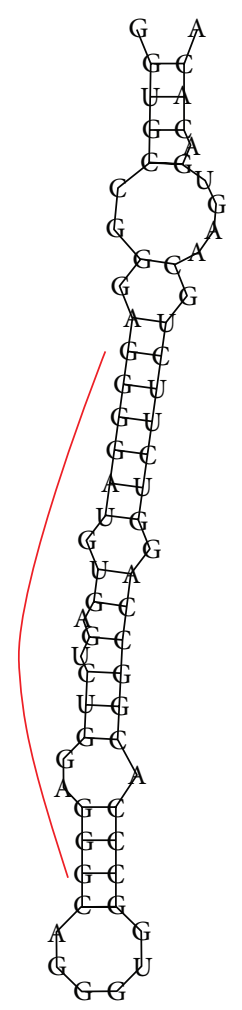

(a)

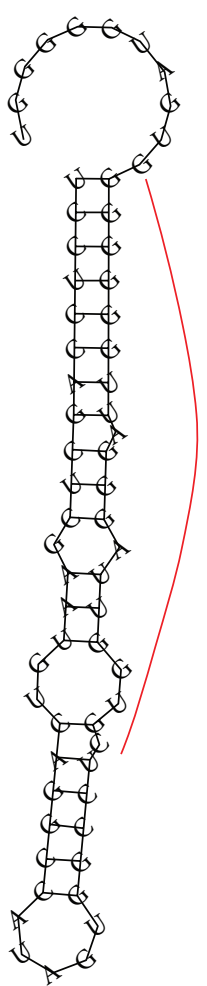

(b)

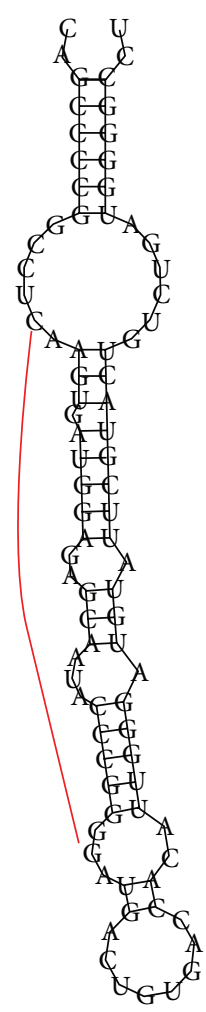

(c)

FIgURE 1: Predicted stem-loop structures of precursors. These 3 precursors had the ability to be processed to form the 3 novel miRNAs in Table 2. The red curve indicated the location that could be processed to miRNAs. (a), (b), and (c) represent tumor, adjacent, and normal control tissues, respectively.

TABLE 1: The numbers of detected miRNAs in 3 pooled samples.

\begin{tabular}{lcc}
\hline Sample & Known miRNAs & Novel miRNAs \\
\hline AC tumor & 259 & 65 \\
AC adjacent & 401 & 95 \\
AC normal & 389 & 34 \\
\hline
\end{tabular}

average $89.72 \%$ reads, were mapped to the reference human genome (data not shown).

To confirm the existence of miRNA among our sequencing miRNAs, we performed a prediction of the miRNA precursor hairpin structure. The novel miRNAs which had the highest expression levels in each pooled sample were shown in Table 2. The secondary structure of their precursor (Figure 1) had the characteristic of stem-loop hairpin structure, and mature miRNA was far from loops and bulges.

The biological feature of the reads annotated to miRNAs was analyzed afterwards. The length distributions of all miRNAs in the 3 pooled samples focused on 21, 22, and $23 \mathrm{nt}$. As expected, reads with a length of $22 \mathrm{nt}$ were the most abundant (data not shown). The peak distributions were consistent with the biological feature of mature miRNAs, indicating that mature miRNAs were well enriched during the preparation of miRNA sequencing library. Simultaneously, all the isomiRs were obtained in each pooled sample. Half of the most dominant isomiR sequences had lengths that were different from those of canonical miRNA sequences. Generally, the most dominant isomiR sequence may be longer or shorter than registered miRNA sequence through altering $5^{\prime}$ or $3^{\prime}$ ends, especially for the $3^{\prime}$ ends (data not shown). Figure 2 illustrated all the isoform profiles of the differential miR-21 which were validated in subsequent experiment (see Sections 3.3 and 3.4).

3.2. The Differentially Expressed miRNAs in Tumor, Adjacent, and Normal Tissues. The differential miRNAs were chosen after three procedures. Firstly, we removed the unreliable miRNAs which had low copy number ( $\leq 10$ reads) in paired samples at the same time. Then the $p$ value and FDR value of miRNA reads were calculated, and the miRNAs with FDR < 0.01 were chosen. At last we obtained the applicable miRNAs with 2 -fold or more change. So 86,50 , and 76 miRNAs were differentially expressed with 2 -fold or greater change in tumor versus normal, adjacent versus normal, tumor versus adjacent tissues, respectively. As shown in Figure 3, with stricter cutoff criteria (fold change $>3$ ), 10 (6 upregulated, 4 downregulated), 12 (8 upregulated, 4 downregulated), and 6 (1 upregulated, 5 downregulated) miRNAs were differentially expressed in tumor versus normal, adjacent versus normal, tumor versus adjacent tissues. 
TABLE 2: The novel miRNAs with the highest expression levels in each pooled sample.

\begin{tabular}{|c|c|c|c|c|c|}
\hline Sample & $\begin{array}{c}\text { Novel } \\
\text { miRNA }\end{array}$ & Arm & Sequence of miRNA & $\begin{array}{l}\text { Sequence of most } \\
\text { abundant taq }\end{array}$ & Sequence of precursor \\
\hline AC tumor & $x x x-1-5 p$ & $5 p$ & $\begin{array}{l}\text { GGGGATGTGAGTCTGGAG- } \\
\text { GG }\end{array}$ & $\begin{array}{l}\text { GGGGATGTGAGT- } \\
\text { CTCGAGTG }\end{array}$ & $\begin{array}{l}\text { GGTGCCGGGAGGGGATGTGAGTCT- } \\
\text { GGAGGGCAGGGTGGCCCACGGCCA- } \\
\text { GGTCTTCTGCAAGTGACACA }\end{array}$ \\
\hline $\begin{array}{l}\mathrm{AC} \\
\text { adjacent }\end{array}$ & $x x x-2-3 p$ & $p$ & $\begin{array}{l}\text { GTGGTTAGGGATTGGGGG- } \\
\text { GG }\end{array}$ & $\begin{array}{l}\text { GTGGTTAGGGAT- } \\
\text { TCGGCGCT }\end{array}$ & $\begin{array}{c}\text { TCCTCCAGCTCGAATGTCAGGCCATAG- } \\
\text { TGGCCTCGTGGTTAGGGATTGGGGGGG- } \\
\text { TGATGGGGGT }\end{array}$ \\
\hline $\begin{array}{l}\mathrm{AC} \\
\text { normal }\end{array}$ & $x x x-3-5 p$ & $5 \mathrm{p}$ & $\begin{array}{c}\text { CAAGTGATGGAGAGCAAT- } \\
\text { ACCCGG }\end{array}$ & $\begin{array}{l}\text { TCAAGTGATGGA- } \\
\text { GAGCAAT }\end{array}$ & $\begin{array}{l}\text { CAGCCCCGGCCTCAAGTGATGGAGAGC- } \\
\text { AATACCCGGGGATGACTGTGACCACAT- } \\
\text { TGGGATGTATTCGTACTGTCTGATGGG- } \\
\text { GCCT }\end{array}$ \\
\hline
\end{tabular}

TABLE 3: The expression levels of 3 miRNAs in 18 pairs of tumor, adjacent, and normal tissue samples of adenocarcinoma quantified by qRT-PCR.

\begin{tabular}{lcccc}
\hline miRNAs & $\begin{array}{c}\text { Mean } \pm \text { SD } \\
\text { in tumor samples }\end{array}$ & $\begin{array}{c}\text { Mean } \pm \text { SD } \\
\text { in adjacent samples }\end{array}$ & $\begin{array}{c}\text { Mean } \pm \text { SD } \\
\text { in normalue samples }\end{array}$ & $\begin{array}{c}\text { (tumor versus normal, } \\
\text { adjacent versus normal, } \\
\text { and } \\
\text { tumor versus adjacent) }\end{array}$ \\
\hline miR-21-5p & $-1.78 \pm 0.12$ & $0.37 \pm 0.08$ & $<0.001$ \\
miR-196a-5p & $0.47 \pm 0.09$ & $1.83 \pm 0.06$ & $3.44 \pm 0.04$ & $<0.001$ \\
miR-218-5p & $1.25 \pm 0.14$ & $0.35 \pm 0.05$ & $-1.05 \pm 0.09$ & $<0.001$ \\
\hline
\end{tabular}

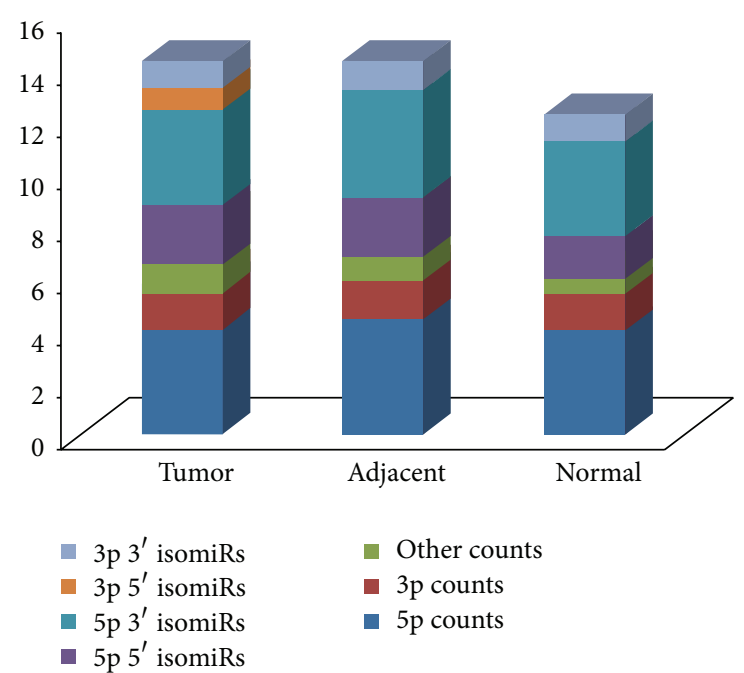

FIGURE 2: All isoform counts of miR-21 are in 3 pooled samples. More $5 \mathrm{p}$ isomiRs and $3^{\prime}$ isomiRs were found in miR-21 compared to $3 p$ isomiRs and $5^{\prime}$ isomiRs, respectively. The $y$-axis meant the value of counts as $\log _{10}$.

3.3. The Gradually Changed miRNAs in Tumor, Adjacent, and Normal Tissues. In this study, we attempted to find out the miRNAs which gradually changed from normal to adjacent to tumor tissues with 2 -fold or greater change and FDR $<0.01$ (the normal as control). As shown in Figure 4(a), there were 61 differential miRNAs in tumor but not in adjacent tissues compared to normal control, 25 miRNAs were expressed differentially in adjacent but not in tumor tissues, 51 miRNAs were differentially expressed in both tumor and adjacent tissues but not in control. In addition, 25 miRNAs were differentially expressed in tumor and adjacent tissues at the same time compared to normal control (Figure 4(b)). Among them, miR-21-5p and miR-196a-5p were gradually upregulated from normal to adjacent to tumor tissues and miR-218-5p was gradually downregulated from normal to adjacent to tumor tissues significantly (Figure 4(c)).

3.4. qRT-PCR Validation of Differential Expression of Gradually Changed miRNAs. In order to validate the differential expression of these 3 gradually changed miRNAs from NGS results, we performed qRT-PCR to quantify the expression levels of miR-21-5p, miR-196a-5p, and miR-218-5p in 18 paired AC samples (including 9 paired AC samples used in miRNA sequencing). The results suggested that miR-21-5p, miR-196a-5p, and miR-218-5p had consistent gradual changes in normal, adjacent, and tumor samples with sequencing results $(p<0.001$, Figure 5$)$, and the magnitude of changes differed between qPCR and NGS. The expression levels of these 3 miRNAs quantified by qPCR were shown in Table 3.

3.5. Prediction and Functional Analysis of Target $m R N A$. The common target genes of these 3 miRNAs were predicted by TargetScan, PicTar, and miRanda based on the starbase. A total of 424 target genes of these 3 differentially expressed miRNAs were predicted and used for GO function enrichment. The significant items of GO biological process, GO 


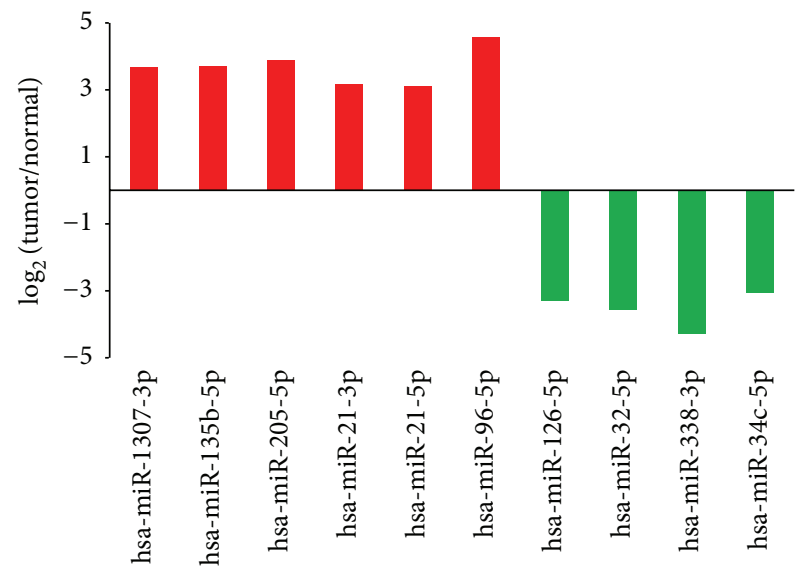

(a)

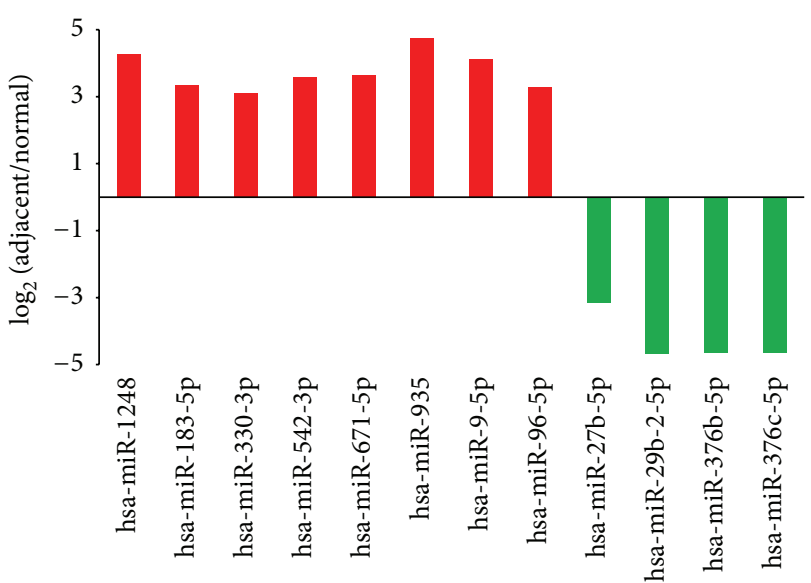

(b)

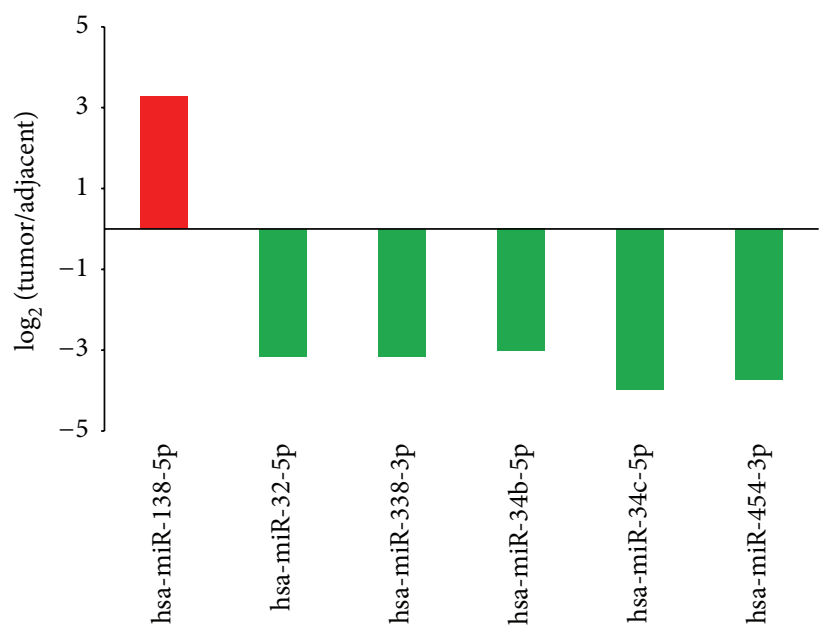

(c)

FIGURE 3: The differential miRNAs with fold change $>3$ in tumor, adjacent, and normal tissues. (a) 10 miRNAs differentially expressed between tumor and normal tissues. (b) 12 miRNAs differentially expressed between adjacent and normal tissues. (c) 6 miRNAs differentially expressed between adjacent and normal tissues. The $y$-axis meant the value of fold change as $\log _{2}$.

molecular function, and GO cell component were shown in Figure 6. We applied the DIANA software to create a rank list of 30 KEGG pathways with the most significant gene enrichment ( $p<0.05$, Table 4). Among them, pathways in cancer ranked first. In addition, plenty of tumor-related pathways were enriched, including non-small-cell lung cancer, colorectal cancer, pancreatic cancer, and prostate cancer. The heatmap of these 3 miRNAs and significant pathways was shown in Figure 7. KEGG pathway showed that miR-215p, miR-196a-5p, miR-218-5p, and their potential target genes were involved in the NSCLC pathway. The results indicated that miR-21-5p, miR-196a-5p, and miR-218-5p might serve as tumor oncogene or suppressor gene in NSCLC (data not shown).

\section{Discussion}

Non-small-cell lung cancer (NSCLC) accounts for $80-85 \%$ of all kinds of lung cancer, the leading cause of cancer deaths worldwide. Adenocarcinoma is one of the major subtypes of NSCLC. miRNAs have been shown to play roles in the tumorigenesis and the development of lung cancer. However, few studies characterize the profiles of miRNAs in paired tumor, adjacent, and normal tissue samples of adenocarcinoma at the same time.

In this study, we found 25 differential miRNAs coexpressed in tumor, adjacent, and normal tissue samples from AC patients based on next-generation sequencing. Among them, 3 miRNAs displayed gradual changes from normal to adjacent to tumor tissues. Then qRT-PCR was performed to confirm that miR-21-5p and miR-196a-5p were gradually upregulated and miR-218-5p was gradually downregulated from normal to adjacent to tumor tissues significantly. The predicted target genes of these 3 miRNAs may regulate in several tumor-related KEGG pathways, including NSCLC, colorectal cancer, pancreatic cancer, and chronic myeloid leukemia. Therefore, pathways in cancer ranked first in all of the enriched KEGG pathways. In particular, miR-21-5p might 


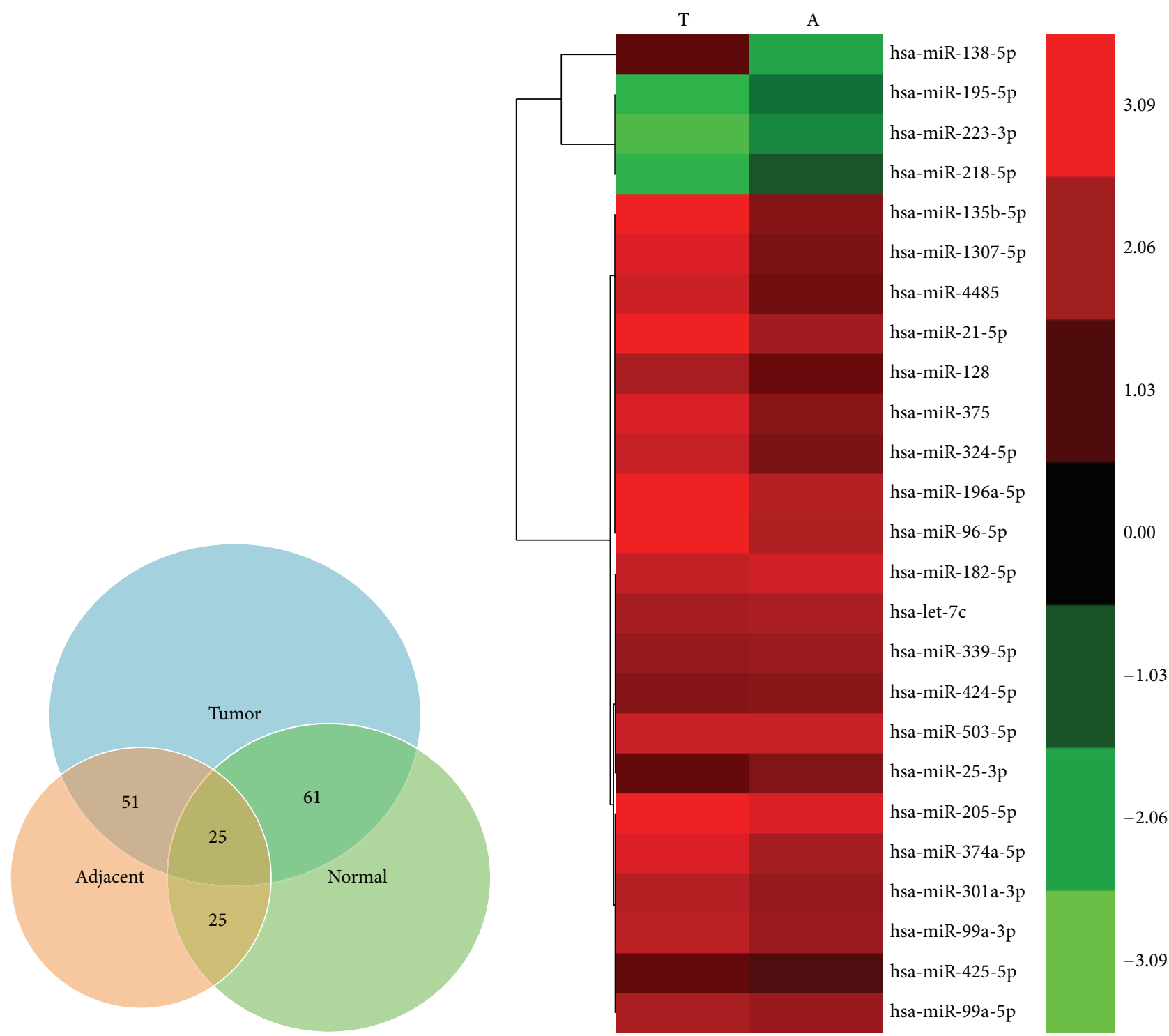

(a)

(b)

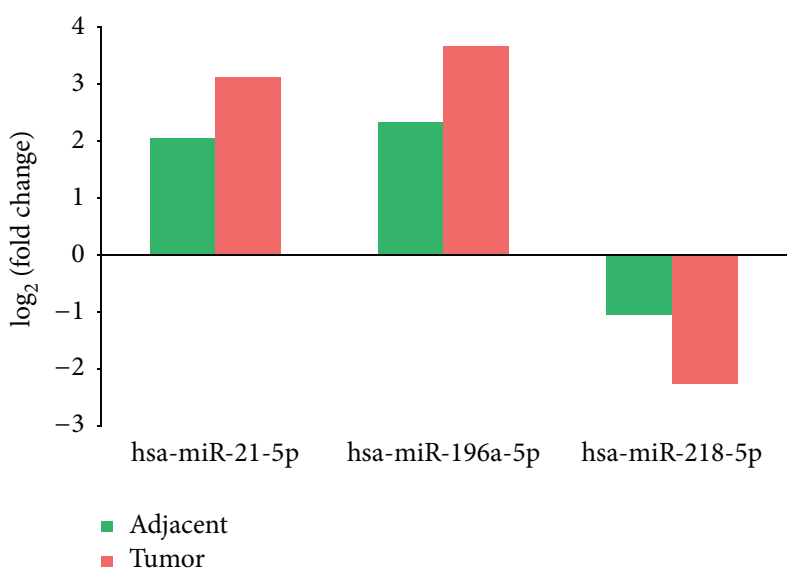

(c)

FIgURE 4: The gradually changed miRNAs with fold change $>2$ in tumor, adjacent, and normal tissues. (a) The sets of differential miRNAs among tumor, adjacent, and normal tissues: 25 miRNAs were differentially expressed in both of tumor and adjacent tissues compared to normal control. (b) Hierarchical clustering analysis of 25 miRNAs dysregulated in tumor and adjacent tissues compared to normal control. The column showed each group compared to the normal control. The lines showed the fold change of each miRNA as $\log _{2}$, from green to red as from -3 to 3 . (c) The 3 miRNAs gradually changed from normal to adjacent to tumor tissues. 
TABLE 4: KEGG pathway of predicted target genes.

\begin{tabular}{|c|c|c|}
\hline KEGG pathway & $\begin{array}{c}\text { Number of } \\
\text { target } \\
\text { genes }\end{array}$ & $p$ value \\
\hline Pathways in cancer & 35 & $6.33 e-17$ \\
\hline Small-cell lung cancer & 15 & $1.04 e-11$ \\
\hline Hepatitis B & 19 & $6.85 e-11$ \\
\hline Colorectal cancer & 11 & $9.08 e-10$ \\
\hline Pancreatic cancer & 13 & $1.57 e-09$ \\
\hline Prostate cancer & 12 & $1.50 e-07$ \\
\hline p53 signaling pathway & 11 & $1.71 e-07$ \\
\hline Bladder cancer & 8 & $7.08 e-07$ \\
\hline Chronic myeloid leukemia & 11 & $8.30 e-07$ \\
\hline PI3K-Akt signaling pathway & 25 & $2.21 e-06$ \\
\hline HIF-1 signaling pathway & 13 & $2.45 e-06$ \\
\hline Focal adhesion & 17 & $6.38 e-05$ \\
\hline mTOR signaling pathway & 8 & 0.00020 \\
\hline $\begin{array}{l}\text { Transcriptional misregulation in } \\
\text { cancer }\end{array}$ & 14 & 0.00034 \\
\hline Endometrial cancer & 7 & 0.00040 \\
\hline Cell cycle & 13 & 0.00069 \\
\hline Melanoma & 8 & 0.00113 \\
\hline $\begin{array}{l}\text { Valine, leucine, and isoleucine } \\
\text { biosynthesis }\end{array}$ & 1 & 0.00337 \\
\hline Glioma & 7 & 0.00337 \\
\hline ErbB signaling pathway & 6 & 0.00337 \\
\hline TGF-beta signaling pathway & 9 & 0.00347 \\
\hline Acute myeloid leukemia & 6 & 0.00406 \\
\hline Amoebiasis & 9 & 0.00846 \\
\hline $\begin{array}{l}\text { Synthesis and degradation of } \\
\text { ketone bodies }\end{array}$ & 2 & 0.01976 \\
\hline Wnt signaling pathway & 11 & 0.01987 \\
\hline Non-small-cell lung cancer & 6 & 0.02290 \\
\hline ECM-receptor interaction & 7 & 0.02443 \\
\hline $\begin{array}{l}\text { Chagas disease (American } \\
\text { trypanosomiasis) }\end{array}$ & 8 & 0.02446 \\
\hline Herpes simplex infection & 13 & 0.02446 \\
\hline $\begin{array}{l}\text { Hypertrophic cardiomyopathy } \\
\text { (HCM) }\end{array}$ & 7 & 0.02446 \\
\hline
\end{tabular}

target several genes in NSCLC pathway, including EGFR, ERBB2, E2F, CDK4, and CDK6. Moreover, miR-21-5p, miR196a-5p, and miR-218-5p were also involved in ErbB signaling pathway, PI3K-Akt signaling pathway, and cell cycle.

For the first time we have characterized the miRNA profiles in tumor, adjacent, and normal tissues from

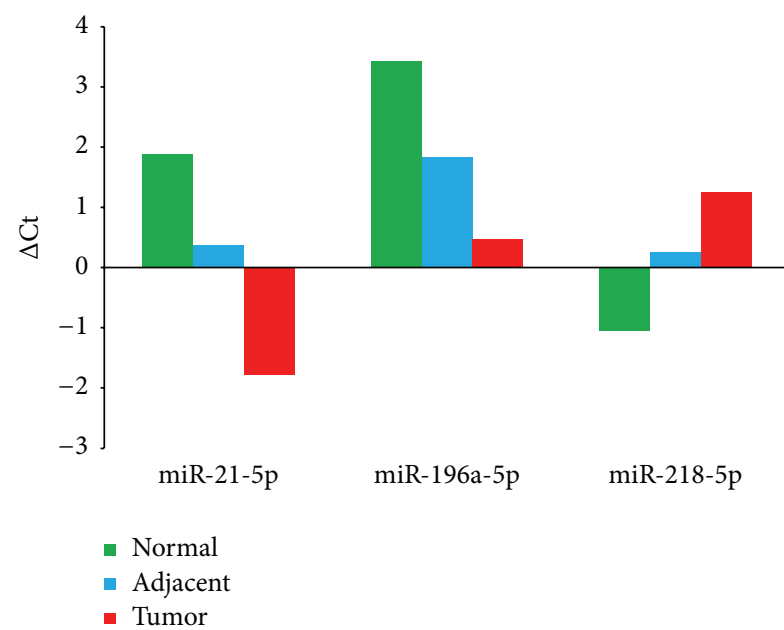

FIGURE 5: The qRT-PCR results of 3 gradually changed miRNAs. miR-21-5p and miR-196a-5p were gradually upregulated from normal to adjacent to tumor tissues, and miR-218-5p was gradually downregulated from normal to adjacent to tumor tissues significantly $\left(p<0.01, \Delta \mathrm{Ct}=\operatorname{AvgCt}_{\mathrm{miRNA}}-\operatorname{AvgCt}_{\mathrm{U} 6}\right)$.

adenocarcinoma patients at the same time. The results showed and validated that miR-21-5p and miR-196a-5p were gradually upregulated, and miR-218-5p was gradually downregulated from normal to adjacent to tumor tissues. These indicated that miR-21-5p and miR-196a-5p might serve as oncogene, and miR-218-5p might act as tumor suppressor gene in lung cancer. However, we need to increase the depth of sequencing to validate the results and gain more data of miRNAs in the future study.

miR-21-5p was comprehensively studied in lung cancer by many researchers. Seike et al. found that aberrantly increased expression of miR-21 was enhanced by activated EGFR signaling pathway and played a significant role in lung carcinogenesis in never-smokers [28]. Markou et al. revealed that mature miRNA-21 was significantly upregulated in NSCLC patients and was an independent negative prognostic factor for overall survival in NSCLC [29]. Xu et al. showed that miR-21 regulated cellular proliferation, invasion, migration, and apoptosis by targeting PTEN, RECK, and Bcl2 in lung squamous carcinoma [30]. However, miR-218-5p and miR-196a-5p were little studied in lung cancer. miR218 was deleted and downregulated in lung squamous cell carcinoma and may be a strong candidate tumor suppressing miRNA potentially involved in lung cancer [31]. Song et al. found that miR-218 suppressed the growth of lung carcinoma by reducing MEF2D expression [32]. Liu et al. showed that miR-196a promoted NSCLC cell proliferation and invasion through targeting HOXA5 [33]. Although the differential expression levels of the 3 miRNAs were approximate to our results in the previous studies, we validated their gradual changes in paired tumor, adjacent, and normal tissues of lung adenocarcinoma for the first time. The gradual changes may verify the function of miR-21-5p, miR-196a-5p, and miR$218-5 p$ as oncogene or suppressor gene more accurately. As little existing research has studied the profiles and functions 


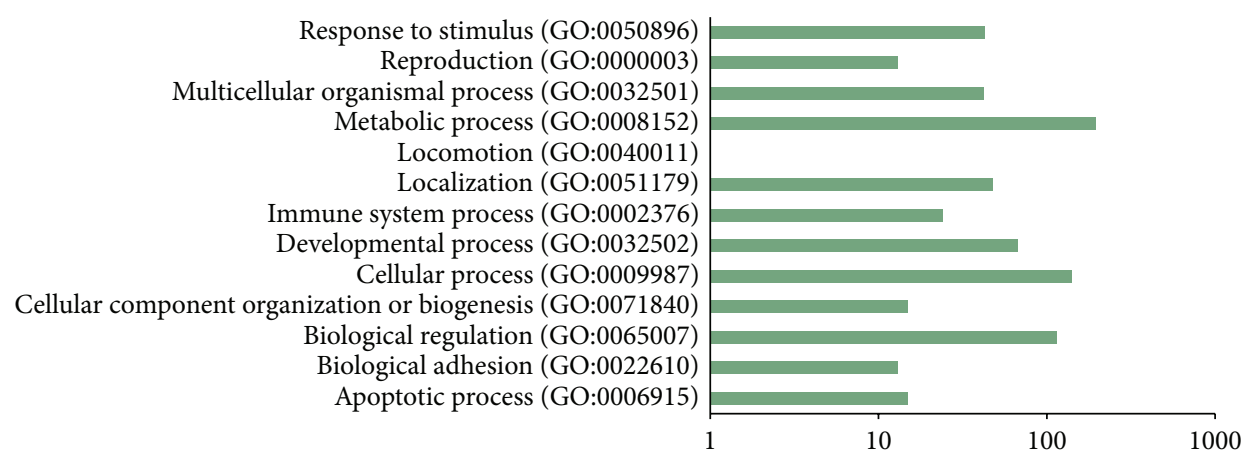

(a)

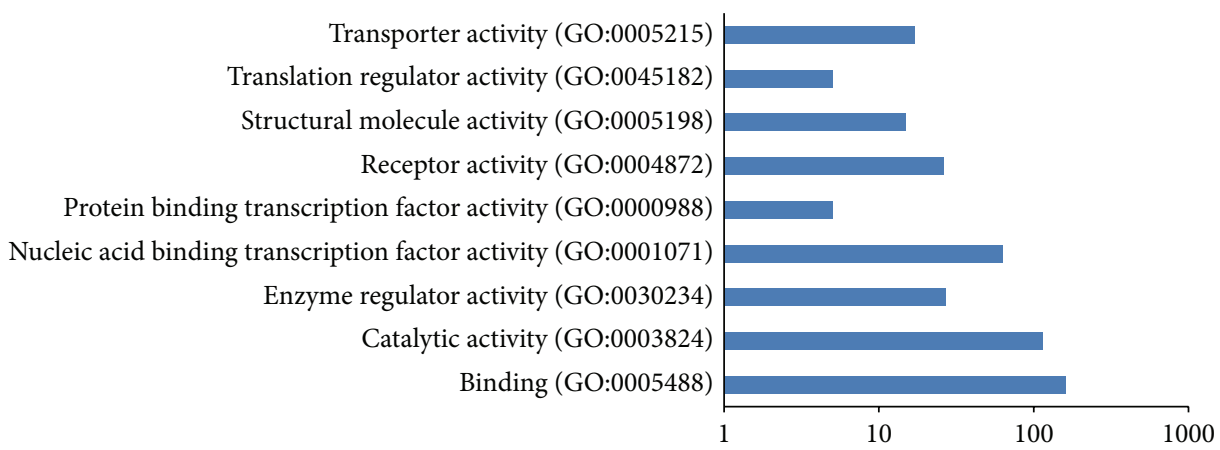

(b)

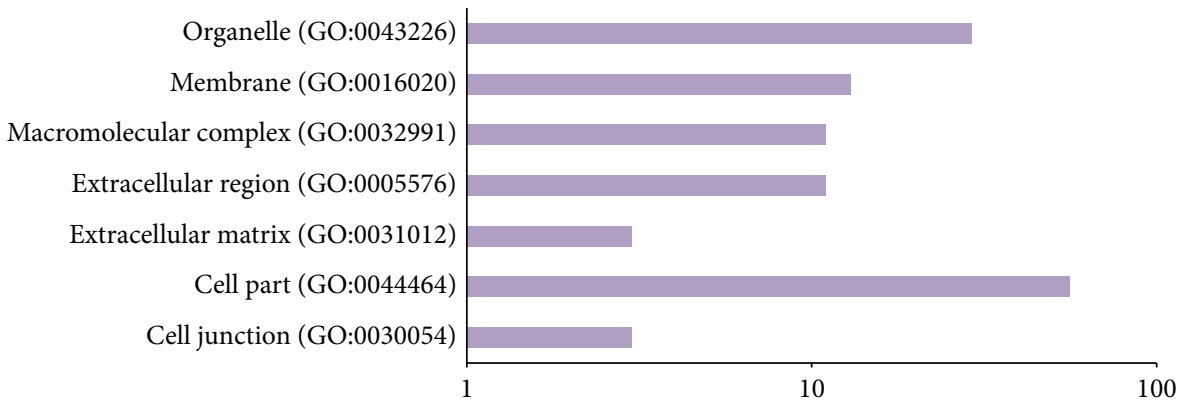

(c)

Figure 6: Categories of the Gene Ontology by target genes of 3 miRNAs. (a) Biological process terms were enriched in metabolic process, cellular process, and biological regulation. (b) Molecular function terms were enriched in binding, catalytic activity, and receptor activity. (c) Cell component terms were enriched in cell part, organelle, and membrane.

of miR-196a-5p and miR-218-5p in lung cancer, we will further validate their regulatory roles in lung cancer in future research.

In conclusion, we characterized the differential expression of miRNAs in paired tumor, adjacent, and normal tissue samples of lung adenocarcinoma based on NGS, and we found and validated the gradual changes of miR-21-5p, miR-196a-5p, and miR-218-5p from normal to adjacent to tumor tissues. These 3 miRNAs may regulate as oncogene or suppressor gene in tumorigenesis and progression of lung adenocarcinoma. Our research provided several miRNA biomarkers for lung adenocarcinoma and may be useful for lung adenocarcinoma diagnosis and the study of pathology in lung cancer.

\section{Competing Interests}

The authors declare no competing interests.

\section{Acknowledgments}

This work was supported by Projects 61271055 and 61372164 of the National Natural Science Foundation of China. 


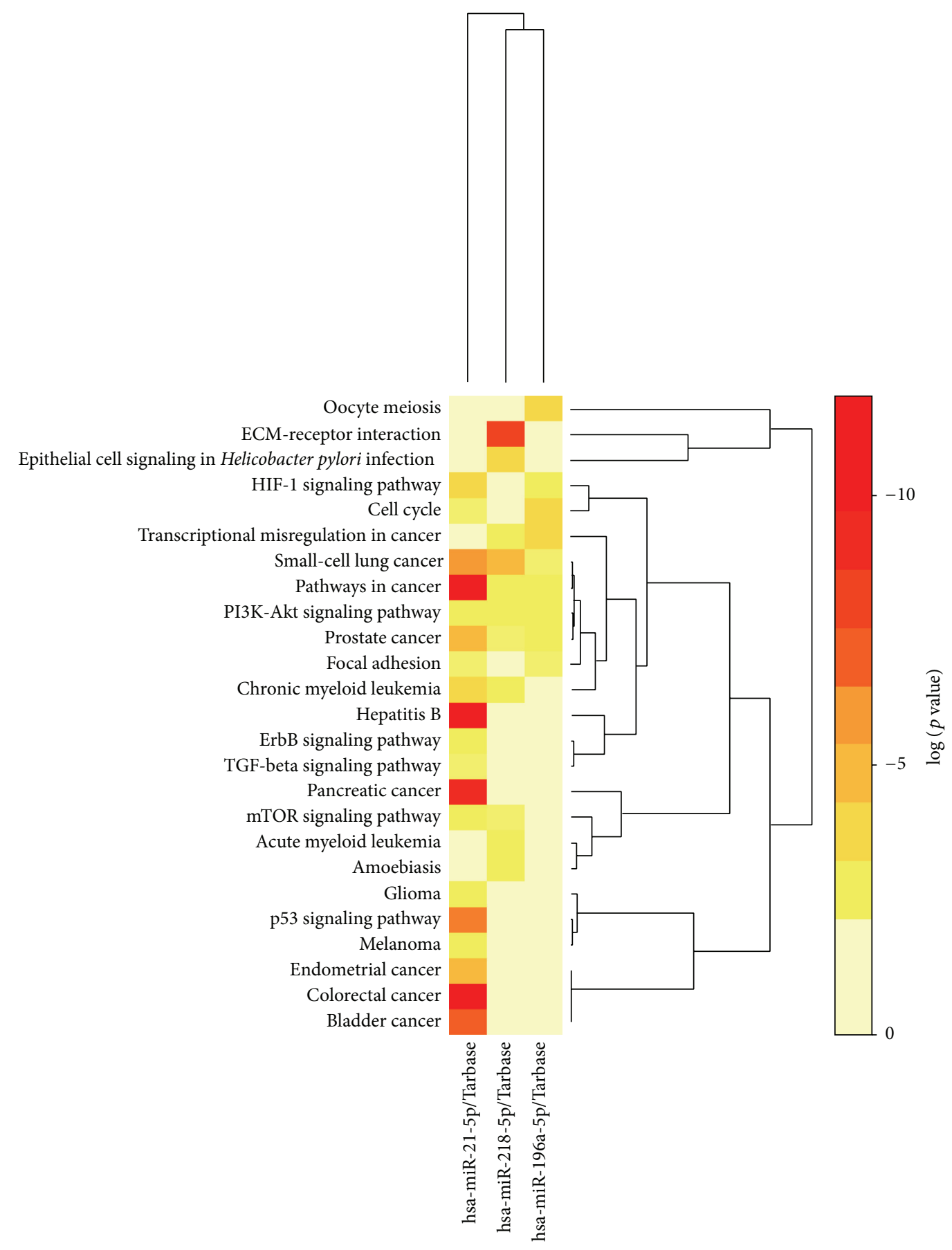

FIGURE 7: The heatmap of significant KEGG pathways that 3 miRNAs may be involved in. The column showed each pathway and candidate target genes of miRNAs. The color showed red to yellow as $\log (p$ value) from low to high.

\section{References}

[1] A. Jemal, R. Siegel, E. Ward, Y. Hao, J. Xu, and M. J. Thun, "Cancer statistics, 2009," CA: A Cancer Journal for Clinicians, vol. 59, no. 4, pp. 225-249, 2009.

[2] T. Maeda, H. Ueoka, M. Tabata et al., "Prognostic factors in advanced non-small cell lung cancer: elevated serum levels of neuron specific enolase indicate poor prognosis," Japanese Journal of Clinical Oncology, vol. 30, no. 12, pp. 534-541, 2000.
[3] A. Jemal, R. Siegel, E. Ward et al., "Cancer statistics, 2008," CA: A Cancer Journal for Clinicians, vol. 58, pp. 71-96, 2008.

[4] S. A. Belinsky, K. J. Nikula, W. A. Palmisano et al., "Aberrant methylation of p16(INK4a) is an early event in lung cancer and a potential biomarker for early diagnosis," Proceedings of the National Academy of Sciences of the United States of America, vol. 95, no. 20, pp. 11891-11896, 1998.

[5] H. Linardou, I. J. Dahabreh, D. Kanaloupiti et al., "Assessment of somatic k-RAS mutations as a mechanism associated with 
resistance to EGFR-targeted agents: a systematic review and meta-analysis of studies in advanced non-small-cell lung cancer and metastatic colorectal cancer," The Lancet Oncology, vol. 9, no. 10, pp. 962-972, 2008.

[6] S. A. Belinsky, "Gene-promoter hypermethylation as a biomarker in lung cancer," Nature Reviews Cancer, vol. 4, no. 9, pp. 707-717, 2004.

[7] D. P. Bartel, "MicroRNAs: genomics, biogenesis, mechanism, and function," Cell, vol. 116, no. 2, pp. 281-297, 2004.

[8] M. A. Valencia-Sanchez, J. Liu, G. J. Hannon, and R. Parker, "Control of translation and mRNA degradation by miRNAs and siRNAs," Genes \& Development, vol. 20, no. 5, pp. 515-524, 2006.

[9] V. Ambros, "MicroRNA pathways in flies and worms: growth, death, fat, stress, and timing," Cell, vol. 113, no. 6, pp. 673-676, 2003.

[10] G. A. Calin and C. M. Croce, "MicroRNA signatures in human cancers," Nature Reviews Cancer, vol. 6, no. 11, pp. 857-866, 2006.

[11] S. P. Nana-Sinkam and C. M. Croce, "Non-coding RNAs in cancer initiation and progression and as novel biomarkers," Molecular Oncology, vol. 5, no. 6, pp. 483-491, 2011.

[12] M. S. Kumar, S. J. Erkeland, R. E. Pester et al., "Suppression of non-small cell lung tumor development by the let-7 microRNA family," Proceedings of the National Academy of Sciences of the United States of America, vol. 105, no. 10, pp. 3903-3908, 2008.

[13] H. Osada and T. Takahashi, "let-7 and miR-17-92: small-sized major players in lung cancer development," Cancer Science, vol. 102, no. 1, pp. 9-17, 2011.

[14] M. Fabbri, R. Garzon, A. Cimmino et al., "MicroRNA-29 family reverts aberrant methylation in lung cancer by targeting DNA methyltransferases 3A and 3B," Proceedings of the National Academy of Sciences of the United States of America, vol. 104, no. 40, pp. 15805-15810, 2007.

[15] A. Git, H. Dvinge, M. Salmon-Divon et al., "Systematic comparison of microarray profiling, real-time PCR, and nextgeneration sequencing technologies for measuring differential microRNA expression," RNA, vol. 16, no. 5, pp. 991-1006, 2010.

[16] P. A. C. 't Hoen, Y. Ariyurek, H. H. Thygesen et al., "Deep sequencing-based expression analysis shows major advances in robustness, resolution and inter-lab portability over five microarray platforms," Nucleic Acids Research, vol. 36, no. 21, article e141, 2008.

[17] A. S. Morrissy, R. D. Morin, A. Delaney et al., "Next-generation tag sequencing for cancer gene expression profiling," Genome Research, vol. 19, no. 10, pp. 1825-1835, 2009.

[18] F. Kuchenbauer, R. D. Morin, B. Argiropoulos et al., "In-depth characterization of the microRNA transcriptome in a leukemia progression model," Genome Research, vol. 18, no. 11, pp. 17871797, 2008.

[19] R. D. Morin, M. D. O'Connor, M. Griffith et al., "Application of massively parallel sequencing to microRNA profiling and discovery in human embryonic stem cells," Genome Research, vol. 18, pp. 610-621, 2008.

[20] J.-H. Li, S. Liu, H. Zhou, L.-H. Qu, and J.-H. Yang, "StarBase v2.0: decoding mirnA-ceRNA, miRNA-ncRNA and proteinRNA interaction networks from large-scale CLIP-Seq data," Nucleic Acids Research, vol. 42, no. 1, pp. D92-D97, 2014.

[21] J. H. Yang, J. H. Li, P. Shao, H. Zhou, Y. Q. Chen, and L. H. Qu, "starBase: a database for exploring microRNA-mRNA interaction maps from Argonaute CLIP-Seq and DegradomeSeq data," Nucleic Acids Research, vol. 39, pp. D202-D209, 2011.
[22] M. Ashburner, C. A. Ball, J. A. Blake et al., "Gene ontology: tool for the unification of biology," Nature Genetics, vol. 25, no. 1, pp. 25-29, 2000.

[23] Gene Ontology Consortium, "Gene ontology consortium: going forward," Nucleic Acids Research, vol. 43, pp. D1049D1056, 2015.

[24] G. L. Papadopoulos, P. Alexiou, M. Maragkakis, M. Reczko, and A. G. Hatzigeorgiou, "DIANA-mirPath: integrating human and mouse microRNAs in pathways," Bioinformatics, vol. 25, no. 15, pp. 1991-1993, 2009.

[25] S. Audic and J.-M. Claverie, "The significance of digital gene expression profiles," Genome Research, vol. 7, no. 10, pp. 986995, 1997.

[26] Y. Benjamini, D. Drai, G. Elmer, N. Kafkafi, and I. Golani, "Controlling the false discovery rate in behavior genetics research," Behavioural Brain Research, vol. 125, no. 1-2, pp. 279-284, 2001.

[27] K. J. Livak and T. D. Schmittgen, "Analysis of relative gene expression data using real-time quantitative PCR and the 2(Delta Delta C(T)) Method," Methods, vol. 25, no. 4, pp. 402-408, 2001.

[28] M. Seike, A. Goto, T. Okano et al., "MiR-21 is an EGFR-regulated anti-apoptotic factor in lung cancer in never-smokers," Proceedings of the National Academy of Sciences of the United States of America, vol. 106, no. 29, pp. 12085-12090, 2009.

[29] A. Markou, E. G. Tsaroucha, L. Kaklamanis, M. Fotinou, V. Georgoulias, and E. S. Lianidou, "Prognostic value of mature microRNA-21 and microRNA-205 overexpression in non-small cell lung cancer by quantitative real-time RT-PCR," Clinical Chemistry, vol. 54, no. 10, pp. 1696-1704, 2008.

[30] L.-F. Xu, Z.-P. Wu, Y. Chen, Q.-S. Zhu, S. Hamidi, and R. Navab, "MicroRNA-21 (miR-21) regulates cellular proliferation, invasion, migration, and apoptosis by targeting PTEN, RECK and Bcl-2 in lung squamous carcinoma, Gejiu City, China," PLoS ONE, vol. 9, no. 8, Article ID e103698, 2014.

[31] M. R. Davidson, J. E. Larsen, I. A. Yang et al., "MicroRNA-218 is deleted and downregulated in lung squamous cell carcinoma," PLoS ONE, vol. 5, no. 9, Article ID e12560, 2010.

[32] L. Song, D. Li, Y. Zhao et al., "miR-218 suppressed the growth of lung carcinoma by reducing MEF2D expression," Tumor Biology, vol. 37, no. 3, pp. 2891-2900, 2016.

[33] X.-H. Liu, K.-H. Lu, K.-M. Wang et al., "MicroRNA-196a promotes non-small cell lung cancer cell proliferation and invasion through targeting HOXA5," BMC Cancer, vol. 12, article 348, 2012. 


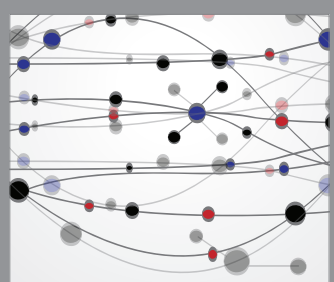

The Scientific World Journal
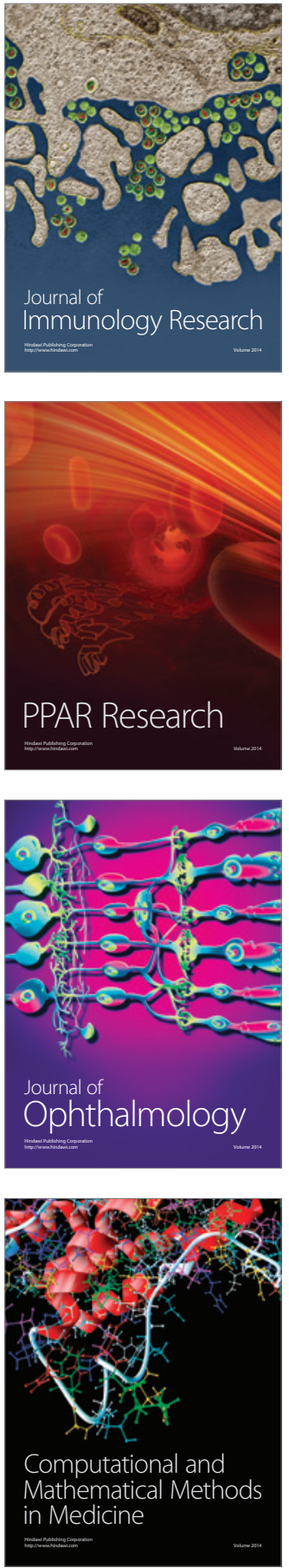

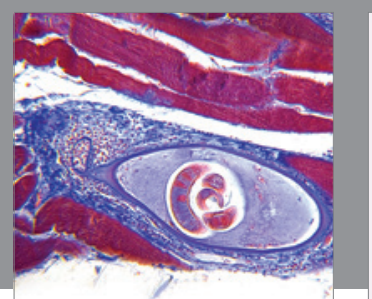

Gastroenterology Research and Practice

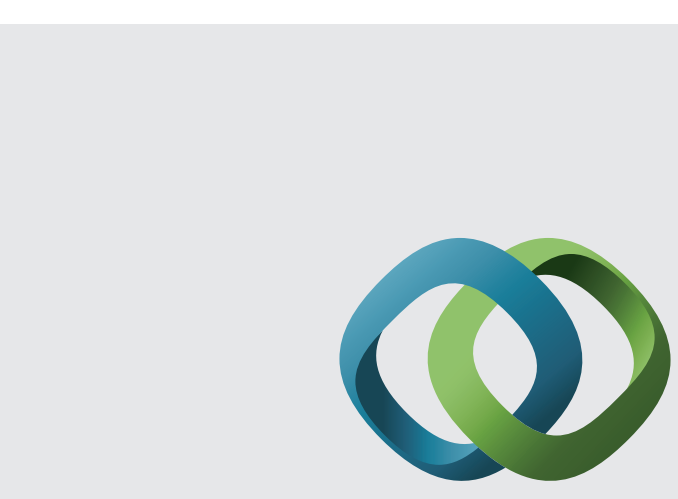

\section{Hindawi}

Submit your manuscripts at

http://www.hindawi.com
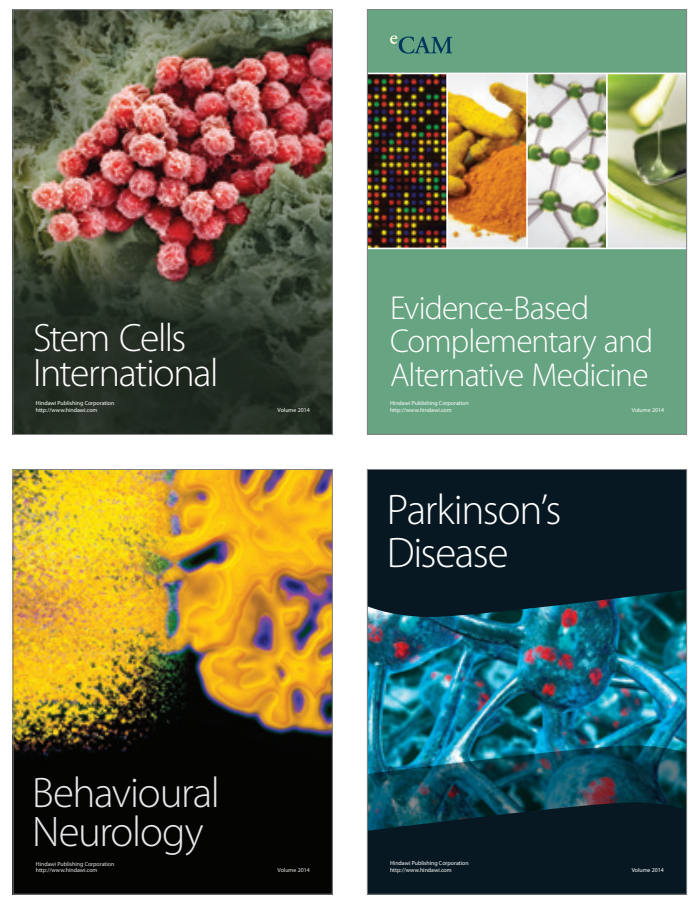
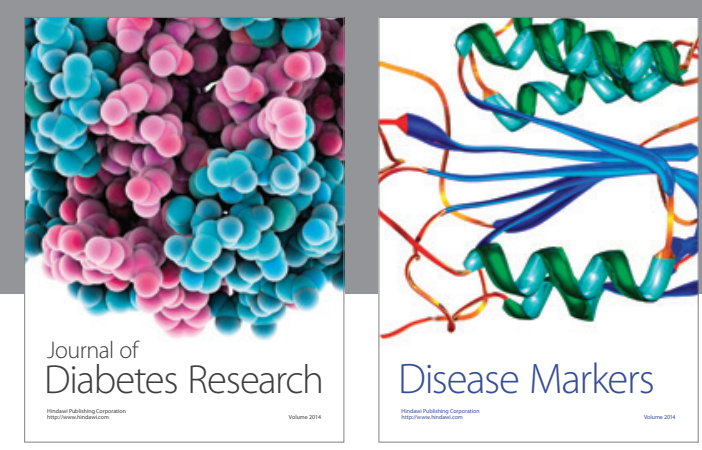

Disease Markers
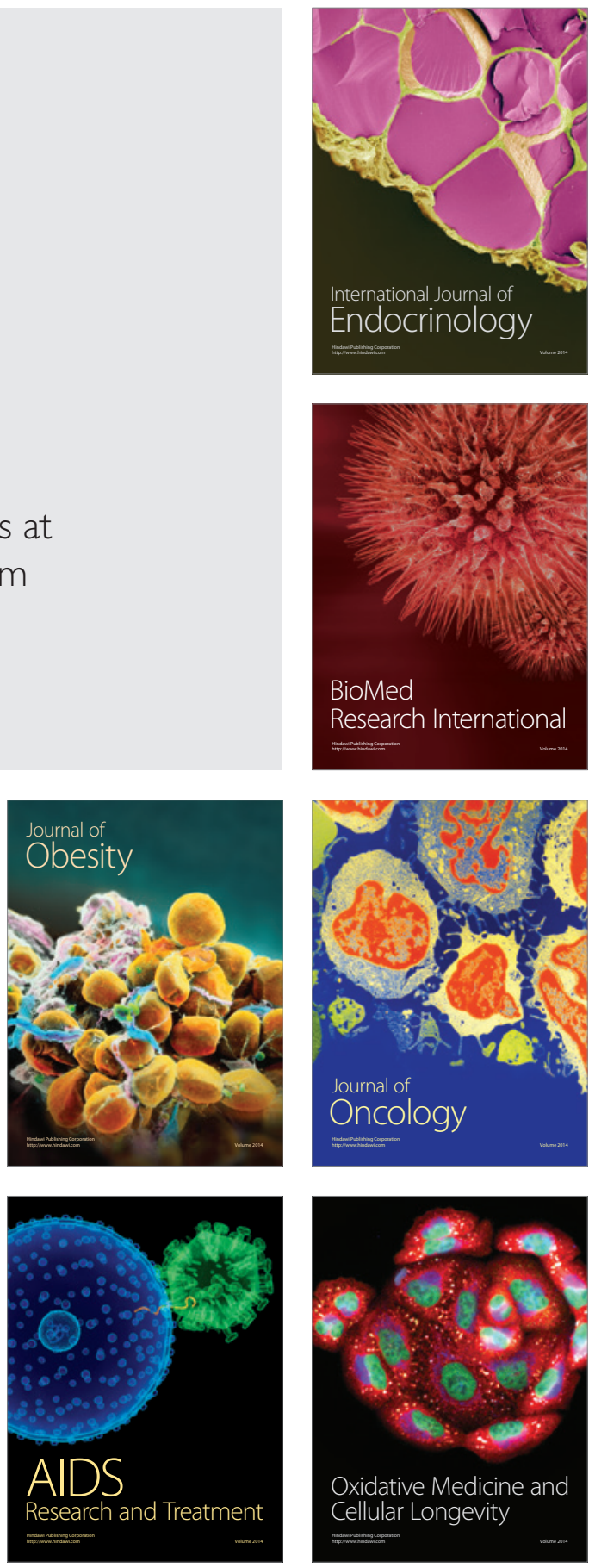